\title{
Adverse events associated with poor neurological outcome during targeted temperature management and advanced critical care after out-of-hospital cardiac arrest
}

Young-Min Kim¹, Chun Song Youn', Soo Hyun Kim², Byung Kook Lee², In Soo Cho ${ }^{3}$, Gyu Chong Cho ${ }^{4}$, Kyung Woon Jeung ${ }^{2}$, Sang Hoon Oh' ${ }^{1}$, Seung Pill Choi ${ }^{1}$, Jong Hwan Shin ${ }^{5}$, Kyoung-Chul Cha ${ }^{6}$, Joo Suk Oh', Hyeon Woo Yim, Kyu Nam Park" ${ }^{7}$, on behalf of the Korean Hypothermia Network Investigators

\begin{abstract}
Introduction: The aim of this study was to investigate the association of adverse events (AEs) during targeted temperature management (TTM) and other AEs and concomitant treatments during the advanced critical care period with poor neurological outcome at hospital discharge in adult out-of-hospital cardiac arrest (OHCA) patients.

Methods: This was a retrospective study using Korean Hypothermia Network registry data of adult OHCA patients treated with TTM in 24 teaching hospitals throughout South Korea from 2007 to 2012. Demographic characteristics, resuscitation and post-resuscitation variables, AEs, and concomitant treatments during TTM and the advanced critical care were collected. The primary outcome was poor neurological outcome, defined as a cerebral performance category (CPC) score of 3-5 at hospital discharge. The AEs and concomitant treatments were individually entered into the best multivariable predictive model of poor neurological outcome to evaluate the associations between each variable and outcome.
\end{abstract}

Results: A total of 930 patients, including 704 for whom a complete dataset of AEs and covariates was available for multivariable modeling, were included in the analysis; 476 of these patients exhibited poor neurological outcome [CPC $3=50(7.1 \%), C P C 4=214(30.4 \%)$, and CPC $5=212(30.1 \%)]$. Common AEs included hyperglycemia (45.6 \%), hypokalemia (31.3\%), arrhythmia (21.3\%) and hypotension (29\%) during cooling, and hypotension (21.6\%) during rewarming. Bleeding (5\%) during TTM was a rare AE. Common AEs during the advanced critical care included pneumonia (39.6\%), myoclonus (21.9\%), seizures (21.7\%) and hypoglycemia within 72 hours (23\%). After adjusting for independent predictors of outcome, cooling- and rewarming-related AEs were not significantly associated with poor neurological outcome. However, sepsis, myoclonus, seizure, hypoglycemia within 72 hours and anticonvulsant use during the advanced critical care were associated with poor neurological outcome [adjusted odds ratios (95\% confidence intervals) of 3.12 (1.40-6.97), 3.72 (1.93-7.16), 4.02 (2.04-7.91), 2.03 (1.09-3.78), and 1.69 (1.03-2.77), respectively]. Alternatively, neuromuscular blocker use was inversely associated with poor neurological outcome (0.48 [0.28-0.84]).

Conclusions: Cooling- and rewarming-related AEs were not associated with poor neurological outcome at hospital discharge. Sepsis, myoclonus, seizure, hypoglycemia within 72 hours and anticonvulsant use during the advanced critical care period were associated with poor neurological outcome at hospital discharge in our study.

\footnotetext{
*Correspondence: emsky@catholic.ac.kr

1 Department of Emergency Medicine, College of Medicine, The Catholic

University of Korea, 222 Banpo-daero, Seocho-gu, Seoul 137-701, South Korea

Full list of author information is available at the end of the article
} 


\section{Introduction}

Out-of-hospital cardiac arrest (OHCA) is an important public health problem in industrialized countries. In the United States, Europe, and South Korea, the estimated incidence of emergency medical services (EMS)-treated OHCA among individuals of any age is 5.7, 38, and 32.7 per 100,000 persons, respectively, and the median rate of survival to hospital discharge is $10.6 \%, 10.7 \%$, and $3.7 \%$, respectively [1-3]. One of the worst complications of sudden cardiac arrest is hypoxic ischemic encephalopathy. Hypoxic ischemic encephalopathy after cardiac arrest results in death or disability in $50 \%$ of patients who exhibit restoration of a pulse $[4,5]$. Since positive results of a quasi-randomized controlled trial and a randomized controlled trial were reported in 2002, targeted temperature management (TTM) has been accepted as the only intervention shown in the last decade to improve outcomes for patients resuscitated from OHCA [6, 7]. Following these landmark studies, many observational studies have reported the effectiveness of TTM, and TTM is recommended as a component of routine post-cardiac arrest care in the international guidelines and a consensus report of five critical care societies [8-10]. Recently, well-performed, large randomized controlled trials also highlighted that targeting a temperature of $36^{\circ} \mathrm{C}$ gives equal results as targeting a temperature of $33^{\circ} \mathrm{C}$ [11-13].

Induced hypothermia may cause various physiological changes as well as adverse events (AEs) [14, 15]. Although AEs commonly occur during TTM and the advanced critical care period, the incidence of most AEs is not significantly different between induced hypothermia and normothermia treatment $[16,17]$. Although some investigators have reported an association between AEs recorded during critical care and mortality, few studies have investigated the relationship between AEs and neurological outcomes in patients with return of spontaneous circulation (ROSC) after OHCA [18-20]. Furthermore, most previous studies have been performed in Western countries, which have relatively high bystander cardiopulmonary resuscitation (CPR) rates and wellestablished EMS systems and where the withdrawal of life-sustaining treatment (WLST) is permitted according to the legislation.

The aim of this study was to investigate the association of cooling-related AEs, rewarming-related AEs, other AEs, and concomitant treatments during the advanced critical care period with poor neurological outcome at the time of hospital discharge in adult OHCA patients who received TTM in South Korea; the baseline characteristics of South Korean adult OHCA patients are different from those of adult OHCA patients in Western countries, and WLST based on prognostication is not yet permitted in South Korea.

\section{Materials and methods Patients}

This was a multicenter, retrospective, registry-based study. This study used the Korean Hypothermia Network (KORHN) registry data. The KORHN, a multicenter clinical research consortium for TTM in South Korea, was organized in 2011 and performed the multicenter retrospective registry project in 2012. The KORHN investigators retrospectively collected data from OHCA patients treated with TTM and advanced critical care at 24 teaching hospitals throughout South Korea from 2007 to 2012. The 24 centers used their own protocol for TTM, but the protocols of most centers included the same parameters of TTM (target temperature of $33^{\circ} \mathrm{C}$, 24-hour maintenance, and controlled normothermia for 72 hours after ROSC). The institutional review board of each institution approved the study protocol before data collection. The name of ethics bodies and site principal investigators are listed in the Acknowledgements section. The institutional review boards waived the requirement to obtain informed consent due to the retrospective nature of the study.

Adult ( $\geq 18$ years) OHCA patients treated with TTM after ROSC were included in the study. Traumatic cardiac arrest patients were excluded. The case report form, standard definitions of 87 variables, and an investigator manual were developed based on a literature review and a consensus of the study investigators (see Additional file 1). The definitions and time points of the variables followed the Utstein recommendations [21, 22]. The registry data were collected from reviews of medical charts or electronic medical records. The data collected from each hospital were verified for completeness by the site principal investigator and were recorded in a web-based data registration system by the site clinical research coordinator. A data manager and three clinical research associates monitored and regularly reviewed data quality. The site principal investigators or site clinical research coordinators were contacted using the query function in the system or directly by phone to clarify data.

\section{Data collection}

We collected data, including demographic characteristics, resuscitation variables, post-resuscitation variables, and AEs and concomitant treatments during TTM and the advanced critical care period. The detailed definitions of the variables and other items included in the registry are described in an Additional file (see Additional file 2) and were reported in an article describing the KORHN registry project [23]. The AEs were divided into cooling-related, rewarming-related, and other AEs during the advanced critical care period. The considered cooling-related and rewarming-related AEs were those which were commonly reported in the literature and were typically attributed to 
TTM $[14,15]$. The terminology of advanced critical care was derived from the American Heart Association adult immediate post-cardiac arrest care algorithm [8]. The advanced critical care period was defined as days 1-7 after ROSC, which was the definition used in previous AE studies [11, 24]. We have included the period as a figure form in a practical manual of post-cardiac arrest care for hospital providers developed by the KORHN. We added the figure in an Additional file (see Figure S1 in Additional file 3).

Overcooling $\left(<32{ }^{\circ} \mathrm{C}\right)$, bradycardia $(<40$ beats $/ \mathrm{min})$, tachyarrhythmia, hypokalemia $(<3.0 \mathrm{mEq} / \mathrm{L})$, hyperglycemia ( $>180 \mathrm{mg} / \mathrm{dL}$ ), bleeding, and hypotension were collected as cooling-related AEs. Rebound hyperthermia (>38 ${ }^{\circ} \mathrm{C}$ within 24 hours of the cessation of cooling), arrhythmia, hyperkalemia $(>5.0 \mathrm{mEq} / \mathrm{L})$, hypoglycemia $(<80 \mathrm{mg} / \mathrm{dL})$, bleeding, and hypotension were collected as rewarming-related AEs. Tachyarrhythmia during cooling was defined as newly developed tachyarrhythmia during cooling except for sinus tachycardia. Bleeding was defined as any site of bleeding associated with cooling and rewarming. Hypotension was defined as systolic blood pressure $(\mathrm{SBP})<90 \mathrm{mmHg}$ or mean arterial pressure (MAP) $<60 \mathrm{mmHg}$ for at least $30 \mathrm{~min}$ or requiring supportive measures (fluid loading and treatment with a vasopressor or inotropic drug or both types of drugs) to maintain the SBP $>90 \mathrm{mmHg}$ or the MAP $>60 \mathrm{mmHg}$ during cooling and rewarming.

Sepsis, pneumonia, myoclonus and seizure occurring during the advanced critical care period that could not be clearly categorized as typical cooling-related or rewarming-related AEs were categorized as other AEs. All hypoglycemic events (defined as $<80 \mathrm{mg} / \mathrm{dL}$ within 72 hours after ROSC and collected in the registry) occurring during the advanced critical care period were categorized as other AEs. The use of analgesics, sedatives, neuromuscular blockers (NMBs), anticonvulsants and insulin during TTM and the advanced critical care period was categorized as concomitant treatment. Sepsis was defined as a clinical syndrome defined by the presence of both documented or suspected infection and systemic inflammatory response syndrome; these cases were categorized as sepsis, severe sepsis, or septic shock [25]. Pneumonia was diagnosed based on the following four clinical criteria without microbiological confirmation: new or progressive consolidation on a chest radiograph, fever, leukocytosis, and the presence of purulent tracheobronchial secretions [19]. Seizure was diagnosed based on either clinically involuntary movements with epileptiform discharges on electroencephalography (EEG) or epileptiform discharges alone on EEG. Myoclonus was defined as clinically involuntary movement without epileptiform discharges on EEG.

\section{Outcome measures}

The goal of our study was to examine the association between AEs and poor neurological outcome at hospital discharge. Poor neurological outcome was defined as a cerebral performance category (CPC) scale score of 3-5. Based on the traditional criteria, CPC 3 indicates severe cerebral disability (conscious but disabled and dependent), CPC 4 indicates coma or a vegetative state (unconscious), and CPC 5 indicates brain death or death [26]. The CPC scores at hospital discharge, which were recorded in the medical charts and the electronic medical records by attending physicians or independent neurologists, were collected. If the score was not recorded, site investigators abstracted these data from the medical records by consensus.

\section{Statistical analysis}

Continuous variables are expressed as the means and standard deviations or the medians and interquartile ranges according to normal distributions, and categorical variables are expressed as numbers and percentages. We used the Wilcoxon rank sum test or the $t$ test and the chi-squared test as appropriate for univariate comparisons of the baseline characteristics between patients with good and poor neurological outcome. First, we determined the clinical variables that were independently associated with functional outcome. Second, we determined the association of cooling-related AEs, rewarming-related AEs, and other AEs during the advanced critical care period with functional outcome, adjusting for the clinical variables identified during the first step.

A multivariate analysis of the relationship between functional outcome and the baseline characteristics was performed using a stepwise logistical regression method, which utilizes a combination of forward and backward selection for multivariable predictive modeling. Variables displaying $p \leq 0.15$ based on univariate analysis were considered as candidates for inclusion in the multivariable predictive model. Continuous variables entered into the model included age (in years), time from arrest to ROSC (in minutes), time from arrest to initiation of cooling (in minutes), time from arrest to $34{ }^{\circ} \mathrm{C}$ (in minutes), and rewarming time (in minutes). Dichotomous variables included sex (male as reference), previously unhealthy (absence of the listed diseases as reference), underlying disease (absence of disease as reference), unwitnessed arrest (witnessed as reference), no bystander CPR (bystander CPR as reference), non-shockable initial rhythm (ventricular fibrillation/pulseless ventricular tachycardia as reference), non-cardiac cause of arrest (cardiac cause of arrest as reference), initial Glasgow coma scale (GCS) score ( $<5$ as reference), initial presence of the pupillary light reflex (absence of the pupillary light reflex as reference), ST-segment elevation myocardial infarction (STEMI) 
(non-STEMI as reference), cardiogenic shock (no cardiogenic shock as reference), and hospital interventions (no intervention as reference). The results are presented as the mean or median differences with $p$ values or odds ratios (ORs) with $95 \%$ confidence intervals (CIs). The goodness-of-fit of the final model was assessed using the Hosmer-Lemeshow test with chi-square analysis and max-rescaled $R$ [2]. To assess the predictiveness of the final model, we calculated the area under the receiver operating characteristic curve (AUC). As a sensitivity analysis, we assessed the adequacy of the final model including all available data from 930 subjects.

The AEs and the concomitant treatments were individually entered into the multivariable predictive model adjusting for the confounders of outcome to evaluate their association with functional outcome. The results are presented as adjusted ORs with $95 \%$ CIs. For exploratory analyses, we performed univariate logistic regression analyses to evaluate the association between each $\mathrm{AE}$ and the clinical variables. Additionally, we performed a sensitivity analysis using the data from the patients who survived until sedation was withdrawn (approximately 3 days after ROSC) to focus on the association between the AEs and functional outcome among these patients. The analyses were conducted using SAS version 9.2 (SAS Institute Inc., Cary, NC, USA). Two-tailed tests of significance were used, and $p<0.05$ was considered to be statistically significant in the analyses.

\section{Results}

\section{Baseline patient characteristics and univariate comparisons of the characteristics}

A total of 930 patients from 24 hospitals were included in the registry. The geographic distribution and the number of patients enrolled in the 24 participating centers were reported as a figure form in an article describing the KORHN registry project [23]. We added the figure in an Additional file (see Figure S2 in Additional file 3). Among these patients, 226 patients for whom data were missing were excluded; as a result, 704 patients were included in the analysis (Fig. 1). Table 1 provides the baseline characteristics, including demographics, resuscitation variables, and post-resuscitation variables, of the included patients and the univariate comparisons of these characteristics between those with good and poor neurological outcome. Of the 228 $(32.4 \%)$ patients with good neurological outcome, $193(27.4 \%)$ and $35(4.9 \%)$ patients were discharged with CPC 1 and 2, respectively. Among the 476 (67.6\%) patients with poor neurological outcome, 50 (7.1\%), $214(30.4 \%)$, and $212(30.1 \%)$ exhibited CPC of 3, 4, and 5, respectively. In our study, surviving OHCA patients were discharged after an average of 14-15 days at the hospital.

\section{Multivariable predictive modeling of poor neurological outcome}

The best multivariable model for predicting poor neurological outcome at the time of hospital discharge included nine independent variables (Table 2). The final model showed the following characteristics: a Hosmer-Lemeshow test: chi-square $=13.6, p=0.093$; max-rescaled $\mathrm{R}[2]=0.659$; and $\mathrm{AUC}=0.929$. Based on a sensitivity analysis of 882 subjects for whom data for the variables were available, the final model also performed well (Hosmer-Lemeshow test: chi-square $=13.6, p=0.714 ;$ max-rescaled $\mathrm{R}[2]=0.655$; and $\mathrm{AUC}=0.933$ ).

\section{Adverse events associated with poor neurological outcome}

The frequencies of TTM-related (cooling- and rewarmingrelated), and other AEs during the advanced critical care period and concomitant treatments during TTM and the advanced critical care period, along with corresponding odds ratios for poor neurological outcome, are presented in Table 3. Table 4 shows the factors associated with selected AEs based on univariate analysis.

\section{TTM-related AEs}

Overcooling, hyperglycemia, and hypotension during the cooling period and hyperkalemia and hypotension during the rewarming period were more frequently observed in patients with poor neurological outcome than in patients with good neurological outcome. However, after adjusting for independent predictors of poor neurological outcome, these AEs were not significantly associated with poor neurological outcome.

Newly developed arrhythmia during TTM was observed in $192(27.3 \%)$ patients and most frequently developed during the cooling period. Bradycardia was more frequently observed in patients with good neurological outcome based on univariate analysis. However, after adjusting for independent predictors of poor neurological outcome, bradycardia was not significantly associated with poor neurological outcome.

Bleeding developed in 35 patients (5\%) during TTM, and 17 patients $(2.4 \%)$ required a transfusion. Among these patients, cooling was stopped due to severe bleeding for only 1 patient $(0.1 \%)$. Based on univariate analysis, intra-aortic balloon pump (IABP) use, continuous renal replacement therapy (CRRT), and cardiogenic shock were associated with bleeding. There was no significant difference in the incidence of bleeding between patients with good and poor neurological outcome at hospital discharge.

\section{Other AEs during the advanced critical care period}

Pneumonia and sepsis were associated with poor neurological outcome based on univariate analysis. After 


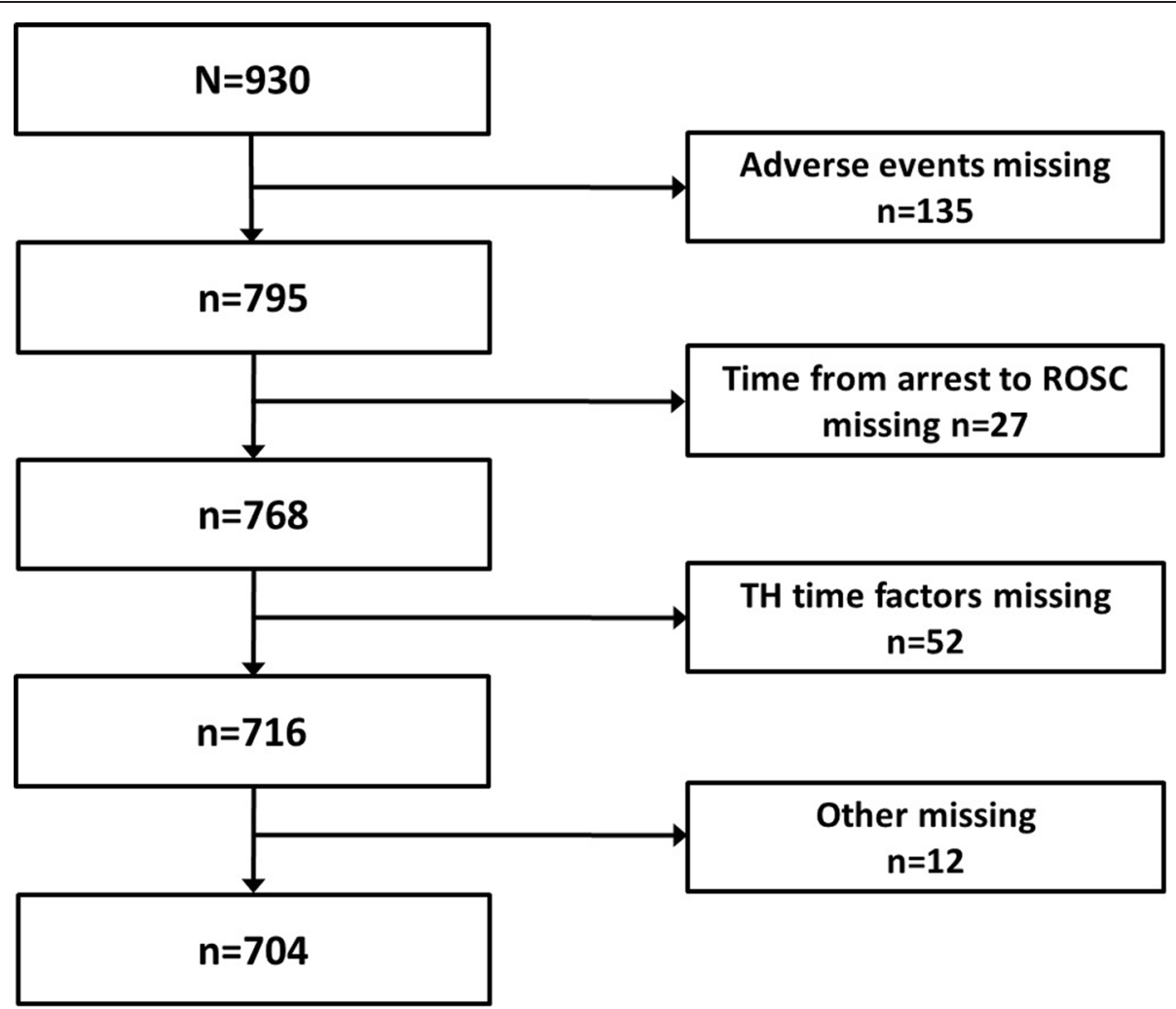

Fig. 1 Flow diagram of the patient selection process

adjusting for independent predictors of poor neurological outcome, sepsis, but not pneumonia, was significantly associated with poor neurological outcome.

Myoclonus, seizure, and hypoglycemia within 72 hours after ROSC were associated with poor neurological outcome based on univariate analysis. After adjusting for the independent predictors of poor neurological outcome, myoclonus, seizure, and hypoglycemia within 72 hours after ROSC remained significantly associated with poor neurological outcome.

\section{Concomitant treatment during TTM and the advanced critical care period}

Patients with poor neurological outcome were more frequently treated with anticonvulsants or insulin than those with good neurological outcome. After adjusting for independent predictors of poor neurological outcome, anticonvulsant use, but not insulin use, was significantly associated with poor neurological outcome. Alternatively, the use of analgesics, sedatives and NMBs was inversely associated with poor neurological outcome based on univariate analysis. After adjusting for independent predictors of poor neurological outcome, only NMB use was inversely associated with poor neurological outcome.

\section{Sensitivity analysis of the patients who survived after sedation was withdrawn}

A total of 32 patients (4.5\%) died within 3 days after ROSC. Based on sensitivity analysis of the remaining 672 patients, the best multivariable model for predicting poor neurological outcome included the same nine independent variables described above. This model displayed the following characteristics: Hosmer-Lemeshow test: chi-square $=7.5, p=0.487$; max-rescaled $\mathrm{R}[2]=0.668$; and $\mathrm{AUC}=0.930$. The same four AEs and one concomitant treatment were significantly associated with poor neurological outcome at hospital discharge based on univariate and multivariate analyses (see Additional file 4).

\section{Discussion}

In this multicenter, registry-based study of adult OHCA patients treated with TTM, cooling-related or rewarmingrelated AEs were not significantly associated with poor neurological outcome at hospital discharge after adjusting for significant predictors of poor neurological outcome. However, sepsis, myoclonus, seizure, hypoglycemia within 72 hours after ROSC and anticonvulsant use during the advanced critical care period were significantly associated with poor neurological outcome at hospital discharge. Based on sensitivity analysis of the 672 patients who 
Table 1 Baseline patient characteristics

\begin{tabular}{|c|c|c|c|}
\hline Patient characteristics & $\begin{array}{l}\text { Good neurological outcome } \\
\text { (CPC } 1 \text { and 2) } n=228 \text { (32.4) }\end{array}$ & $\begin{array}{l}\text { Poor neurological outcome } \\
(\text { CPC 3-5) } n=476 \text { (67.6) }\end{array}$ & $p$ \\
\hline \multicolumn{4}{|l|}{ Background variables } \\
\hline Age, years & $50(40-60)$ & $59(47-71)$ & $<.001$ \\
\hline Male & 177 (77.6) & $323(67.9)$ & 0.008 \\
\hline Previously healthy ${ }^{a}$ & $130(57.0)$ & $178(37.4)$ & $<.001$ \\
\hline Myocardial infarction & $13(5.7)$ & $26(5.5)$ & 0.897 \\
\hline Angina & $19(8.3)$ & $29(6.1)$ & 0.270 \\
\hline Congestive heart failure & $5(2.2)$ & $19(4.0)$ & 0.219 \\
\hline Hypertension & $57(25.0)$ & $172(36.1)$ & 0.003 \\
\hline Diabetes mellitus & $29(12.7)$ & $116(24.4)$ & $<.001$ \\
\hline Pulmonary disease & $4(1.8)$ & $35(7.4)$ & 0.002 \\
\hline Stroke & $4(1.8)$ & $27(5.7)$ & 0.018 \\
\hline Other neurological disease & $7(3.1)$ & $35(7.4)$ & 0.025 \\
\hline Renal disease & $4(1.8)$ & $37(7.8)$ & 0.001 \\
\hline Liver cirrhosis & $1(0.4)$ & $9(1.9)$ & 0.180 \\
\hline Malignancy & $5(2.2)$ & $14(2.9)$ & 0.567 \\
\hline \multicolumn{4}{|l|}{ Resuscitation variables } \\
\hline Witnessed arrest & $182(79.8)$ & $290(60.9)$ & $<.001$ \\
\hline Bystander CPR & $85(37.3)$ & $121(25.4)$ & 0.001 \\
\hline First monitored rhythm & & & $<.001$ \\
\hline VF/pulseless VT & $148(64.9)$ & $89(18.7)$ & \\
\hline Pulseless electrical activity & $28(12.3)$ & $77(16.2)$ & \\
\hline Asystole & $41(17.9)$ & $299(62.8)$ & \\
\hline Unknown & $11(4.8)$ & $11(2.3)$ & \\
\hline Cardiac cause of arrest & $201(88.2)$ & $232(48.7)$ & $<.001$ \\
\hline Time from arrest to ROSC, min & $24.5(16.5-34.5)$ & $33(25-45)$ & $<.001$ \\
\hline \multicolumn{4}{|l|}{ Post-resuscitation variables } \\
\hline Initial Glasgow coma scale $\geq 5$ & $76(33.3)$ & $23(4.8)$ & $<.001$ \\
\hline Initial presence of pupillary light reflex & $178(78.1)$ & $132(27.7)$ & $<.001$ \\
\hline ST-elevation myocardial infarction & $36(15.8)$ & $26(5.5)$ & $<.001$ \\
\hline Cardiogenic shock ${ }^{\mathrm{b}}$ & $64(28.1)$ & $127(26.7)$ & 0.698 \\
\hline Thrombolysis & $2(0.9)$ & $3(0.6)$ & 0.661 \\
\hline Coronary angiography & $152(66.7)$ & $50(10.5)$ & $<.001$ \\
\hline Percutaneous coronary intervention & $44(19.3)$ & $25(5.3)$ & $<.001$ \\
\hline Coronary artery bypass graft & $7(3.1)$ & $0(0.0)$ & $<.001$ \\
\hline Intra-aortic balloon pump & $8(3.5)$ & $11(2.3)$ & 0.359 \\
\hline Extracorporeal membrane oxygenation & $4(1.8)$ & $10(2.1)$ & 1.000 \\
\hline Continuous renal replacement therapy & $4(1.8)$ & $49(10.3)$ & $<.001$ \\
\hline Time from arrest to initiation of cooling, min & $142(81.5-247.5)$ & $140(80-240)$ & 0.629 \\
\hline Time from arrest to $34^{\circ} \mathrm{C}$, min & $385(257.5-570)$ & $307(200-2454.5)$ & $<.001$ \\
\hline Rewarming time, min & $666(420-780)$ & $720(480-990)$ & 0.002 \\
\hline
\end{tabular}

Data are presented as medians (interquartile range) or counts (percentage)

CPC cerebral performance category, CPR cardiopulmonary resuscitation, $V F$ ventricular fibrillation, $V T$ ventricular tachycardia, $R O S C$ return of spontaneous circulation

${ }^{\mathrm{a}}$ Absence of the listed diseases

${ }^{\mathrm{b}}$ Hypotension (SBP $<90 \mathrm{mmHg}$ or MAP $<60 \mathrm{mmHg}$ for at least $30 \mathrm{~min}$ or the need for supportive measures (fluid loading and vasopressors or inotropic or both) to maintain a SBP $>90 \mathrm{mmHg}$ or MAP $>60 \mathrm{mmHg}$ ) and end-organ hypoperfusion (cool extremities) 
Table 2 Multivariable predictive model for poor neurological outcomes at the time of hospital discharge

\begin{tabular}{|c|c|c|c|}
\hline Variables & OR & $(95 \% \mathrm{Cl})$ & $p$ \\
\hline Continuous renal replacement therapy ${ }^{a}$ & 5.66 & $(1.85-21.97)$ & 0.005 \\
\hline Non-shockable initial rhythm ${ }^{\text {b }}$ & 2.97 & $(1.72-5.16)$ & 0.009 \\
\hline Non-cardiac cause of arrest $^{c}$ & 2.73 & $(1.47-5.17)$ & 0.002 \\
\hline Previously unhealthy ${ }^{d}$ & 2.07 & $(1.20-3.60)$ & 0.001 \\
\hline Time from arrest to ROSC ${ }^{\mathrm{e}}$ & 1.06 & $(1.04-1.08)$ & $<.001$ \\
\hline $\operatorname{Age}^{f}$ & 1.03 & $(1.01-1.05)$ & 0.001 \\
\hline Initial Glasgow coma scale $\geq 5^{\mathrm{g}}$ & 0.29 & $(0.14-0.58)$ & 0.001 \\
\hline Initial presence of pupillary light reflex ${ }^{h}$ & 0.28 & $(0.17-0.46)$ & $<.001$ \\
\hline Coronary angiography ${ }^{i}$ & 0.13 & $(0.14-0.58)$ & $<.001$ \\
\hline
\end{tabular}

OR odds ratio, $95 \%$ Cl $95 \%$ confidence interval, ROSC return of spontaneous circulation

${ }^{a}$ No continuous renal replacement therapy as reference

${ }^{\mathrm{b}} \mathrm{VF} /$ pulseless VT as reference

${ }^{c} \mathrm{Cardiac}$ cause of arrest as reference

${ }^{d}$ Absence of the listed diseases as reference

${ }^{\mathrm{e}}$ By minute

By year

${ }^{\mathrm{g}}<5$ as reference

${ }^{\mathrm{h}}$ Absence of pupillary light reflex as reference

'No coronary angiography as reference

survived after sedation was withdrawn, the results were consistent with the findings of the primary analysis.

To the best of our knowledge, this is the largest observational study to evaluate the association of AEs occurring during TTM and the advanced critical care period with neurological outcome at hospital discharge in adult OHCA patients treated with TTM. Although we were only able to investigate neurological outcome at hospital discharge, the outcomes reported in our study may represent the natural course of OHCA patients treated with TTM in the hospitals because WLST based on prognostication was not applied to these patients.

The results of neurological outcome among our survivors were quite different from those observed in previous studies performed in Western countries [11, 18, 19]. Compared to the results of prospective observational studies by Nielsen et al. [18, 19], in which only a few patients (1\%) exhibited CPC 4, 214 patients (30.4\%) exhibited CPC 4 in our study. In the Target Temperature Management trial, only $19(4 \%)$ patients at hospital discharge and $6(1 \%)$ patients at 6 months had CPC 4 [11]. These differences may result from the difference in baseline patients' characteristics, especially certain resuscitation-related variables, lower rate of WLST in our study population, and difference in end points between two studies (at 6 months versus at hospital discharge). Because prehospital chains are relatively weak in South Korea, resuscitation variables such as witnessed arrest, bystander CPR, and initial rhythm were different in our study compared to previous studies performed in Western countries. In prospective observational studies from
Europe and North America, the proportions of witnessed arrest, bystander CPR, and initial shockable rhythm were higher than those in our study $[11,18,19]$. In contrast to the rate of WLST in Western countries, where WLST is the most common reason for death among cardiac arrest survivors [5, 27], the rate of WLST is very low in South Korea due to local cultural factors. Regardless of neurological status or the results of prognostication tests, most family members choose to continue care for comatose survivors resuscitated from cardiac arrest. Although the Korean Medical Association recommended consensus guidelines in 2009, WLST remains a matter of debate, and WLST for post-cardiac arrest patients based on prognostication is not legally permitted in South Korea. This circumstance provides an excellent opportunity to collect unbiased data on the association between prognostic factors including AEs and ultimate neurological outcomes in the modern intensive care setting. One of the most valuable results of our study is the generation of a multivariable predictive model of poor neurological outcome at the time of hospital discharge. Because this model was fitted to data in which WLST was not performed, our results address a critical limitation of most modern studies from Europe and North America.

Bleeding during TTM was rare in our study, and its incidence was similar to that of most previous reports $[7,11,16-20]$. Bleeding during TTM was more frequently observed in patients receiving an invasive intervention, such as IABP, extracorporeal membrane oxygenation (ECMO), and cardiogenic shock, whereas thrombolysis and the use of an endovascular device, coronary angiography, or percutaneous coronary intervention $(\mathrm{PCI})$ were not associated with an increased risk of bleeding based on univariate analysis. These results do not correspond to the findings of the prospective study by Nielsen et al., in which thrombolysis and the use of an endovascular device were associated with an increased risk of bleeding based on univariate analysis [19]. However, bleeding was not significantly associated with the outcome in either study. These results are consistent with the conclusion of a recent systematic review and meta-analysis of the risk of bleeding [28].

The incidence of newly developed arrhythmia during TTM was slightly lower than that observed in previous studies $[11,19,29,30]$. Because we only retrospectively collected the arrhythmia data during the TTM period, the incidence observed in this study could be lower than that observed by others. Recently, bradycardia during TTM has been suggested to be a predictor of favorable outcome $[29,30]$. In our study, bradycardia during the cooling period more frequently developed in patients with good neurological outcome based on univariate analysis. However, after adjusting for independent predictors of poor neurological outcome, bradycardia was not significantly 
Table 3 Adverse events and concomitant treatments with corresponding odds ratios for poor neurological outcomes at the time of hospital discharge

\begin{tabular}{|c|c|c|c|c|c|c|c|c|}
\hline Category & $\begin{array}{l}\text { AEs and concomitant } \\
\text { treatments }\end{array}$ & Total N (\%) & $\begin{array}{l}\text { CPC } 1 \text { and } 2 \\
\mathrm{n}(\%)\end{array}$ & $\begin{array}{l}\text { CPC 3-5 } \\
\mathrm{n}(\%)\end{array}$ & OR $(95 \% \mathrm{Cl})$ & $p$ & $\begin{array}{l}\text { Adjusted } \\
\text { OR (95 \% Cl) }\end{array}$ & $p$ \\
\hline & & $704(100)$ & $228(32.4)$ & $476(67.6)$ & & & & \\
\hline \multirow[t]{7}{*}{ Cooling -related AEs } & Overcooling ${ }^{a}$ & $128(18.2)$ & $23(10.1)$ & $105(22.1)$ & $2.52(1.56-4.09)$ & $<.001$ & $1.16(0.58-2.31)$ & 0.681 \\
\hline & Bradycardia $^{\mathrm{b}}$ & $83(11.8)$ & $35(15.4)$ & $48(10.1)$ & $0.62(0.39-0.99)$ & 0.044 & $0.75(0.37-1.53)$ & 0.429 \\
\hline & Tachyarrhythmia & $67(9.5)$ & $24(10.5)$ & $43(9.0)$ & $0.84(0.50-1.43)$ & 0.528 & $0.72(0.33-1.55)$ & 0.398 \\
\hline & Hypokalemia $^{c}$ & $220(31.3)$ & $65(28.5)$ & $155(32.6)$ & $1.21(0.86-1.71)$ & 0.278 & $1.21(0.71-2.04)$ & 0.486 \\
\hline & Hyperglycemia $^{d}$ & $321(45.6)$ & $81(35.5)$ & $240(50.4)$ & $1.85(1.33-2.56)$ & $<.001$ & $1.37(0.84-2.24)$ & 0.210 \\
\hline & Bleeding & $21(3.0)$ & $6(2.6)$ & $15(3.2)$ & $1.20(0.46-3.15)$ & 0.705 & $0.89(0.21-3.71)$ & 0.872 \\
\hline & Hypotension $^{e}$ & $204(29.0)$ & $54(23.7)$ & $150(31.5)$ & $1.48(1.03-2.13)$ & 0.033 & $1.03(0.59-1.79)$ & 0.926 \\
\hline \multirow[t]{6}{*}{ Rewarming -related AEs } & Rebound hyperthermia $^{f}$ & $85(12.1)$ & $29(12.7)$ & $56(11.8)$ & $0.92(0.57-1.48)$ & 0.716 & $1.33(0.61-2.92)$ & 0.473 \\
\hline & Arrhythmia & $42(6.0)$ & $10(4.4)$ & $32(6.7)$ & $1.57(0.76-3.25)$ & 0.224 & $1.70(0.61-4.69)$ & 0.308 \\
\hline & Hyperkalemia ${ }^{9}$ & $48(6.8)$ & $7(3.1)$ & 41 (8.6) & $2.96(1.31-6.74)$ & 0.009 & $1.09(0.37-3.19)$ & 0.881 \\
\hline & Hypoglycemiah $^{\mathrm{h}}$ & $70(9.9)$ & $22(9.7)$ & $48(10.7)$ & $1.05(0.62-1.79)$ & 0.858 & $1.14(0.50-2.57)$ & 0.762 \\
\hline & Bleeding & $14(2.0)$ & $3(1.3)$ & $11(2.3)$ & $1.77(0.49-6.41)$ & 0.383 & $1.05(0.18-6.06)$ & 0.956 \\
\hline & Hypotension ${ }^{e}$ & $152(21.6)$ & $36(15.8)$ & $116(24.4)$ & $1.72(1.14-2.60)$ & 0.01 & $0.99(0.54-1.83)$ & 0.984 \\
\hline \multirow{10}{*}{$\begin{array}{l}\text { Other AEs during advanced critical } \\
\text { care Concomitant treatments }\end{array}$} & Pneumonia ${ }^{i}$ & 279 (39.6) & $70(30.7)$ & $209(43.9)$ & $1.77(1.26-2.47)$ & $<.001$ & $1.05(0.63-1.74)$ & 0.852 \\
\hline & Sepsis & $100(14.2)$ & $17(7.5)$ & $83(17.4)$ & $2.62(1.52-4.53)$ & $<.001$ & $3.12(1.40-6.97)$ & 0.005 \\
\hline & Myoclonus j & $154(21.9)$ & $34(14.9)$ & $120(25.2)$ & $1.92(1.27-2.92)$ & 0.002 & $3.72(1.93-7.16)$ & $<.001$ \\
\hline & Seizurek & $153(21.7)$ & $24(10.5)$ & $129(27.1)$ & $3.16(1.98-5.05)$ & $<.001$ & $4.02(2.04-7.91)$ & $<.001$ \\
\hline & Hypoglycemia' & $162(23.0)$ & $36(15.8)$ & $126(26.5)$ & $1.92(1.27-2.89)$ & 0.002 & $2.03(1.09-3.78)$ & 0.025 \\
\hline & Anticonvulsants & $287(40.8)$ & $71(31.1)$ & $216(45.4)$ & $1.84(1.32-2.56)$ & $<.001$ & $1.69(1.03-2.77)$ & 0.039 \\
\hline & Insulin & $460(65.3)$ & 136(59.7) & $324(68.1)$ & $1.44(1.04-2.00)$ & 0.028 & $0.98(0.60-1.62)$ & 0.944 \\
\hline & Analgesics & $323(45.9)$ & $122(53.5)$ & $201(42.2)$ & $0.64(0.46-0.87)$ & 0.005 & $0.75(0.46-1.21)$ & 0.239 \\
\hline & Sedatives & $657(93.3)$ & $222(97.4)$ & 435 (91.4) & $0.29(0.12-0.69)$ & 0.005 & $0.92(0.29-2.96)$ & 0.888 \\
\hline & $\begin{array}{l}\text { Neuromuscular } \\
\text { blockers }\end{array}$ & 475 (67.5) & $174(76.3)$ & 301 (63.2) & $0.53(0.37-0.76)$ & 0.001 & $0.48(0.28-0.84)$ & 0.009 \\
\hline
\end{tabular}

$A E s$ adverse events, $C P C$ cerebral performance category, OR odds ratio, $95 \%$ Cl $95 \%$ confidence interval

${ }^{a}<32{ }^{\circ} \mathrm{C}$

${ }^{\mathrm{b}}$ Heart rate $<40 \mathrm{bpm}$

${ }^{c} \leq 3.0 \mathrm{mEq} / \mathrm{L}$

d $\geq 180 \mathrm{mg} / \mathrm{Dl}$

${ }^{\text {e }} \mathrm{SBP}<90 \mathrm{mmHg}$ or MAP $<60 \mathrm{mmHg}$ for at least $30 \mathrm{~min}$ or the need for supportive measures (fluid loading and vasopressors or inotropic or both) to maintain a $\mathrm{SBP}>90 \mathrm{mmHg}$ or MAP $>60 \mathrm{mmHg}$

${ }^{\mathrm{f}}>38^{\circ} \mathrm{C}$ within 24 hours of the cessation of cooling

${ }^{\mathrm{g}} \geq 5.0 \mathrm{mEq} / \mathrm{L}$

$\mathrm{h} \leq 80 \mathrm{mg} / \mathrm{dL}$

'Four requirements: 1) new or progressive consolidation on the chest radiograph, 2) fever, 3) leukocytosis, and 4) the presence of purulent tracheobronchial secretions

${ }^{j}$ Clinically involuntary movement without epileptiform discharge on EEG

${ }^{k}$ Either clinically involuntary movement with epileptiform discharge on electroencephalogram (EEG) or epileptiform discharge only on EEG

${ }^{\prime} \leq 80 \mathrm{mg} / \mathrm{dL}$ within 72 hours after ROSC

associated with poor neurological outcome at hospital discharge.

Hypotension during TTM was common but was not significantly associated with poor neurological outcome at hospital discharge after adjusting for independent predictors in our study. This finding is consistent with the results of a previous study showing that MAP time integral during TTM were not significantly associated with functional outcome at hospital discharge [31]. However, the impact of hypotension during TTM on functional outcome is controversial. Many studies showed that early hypotension (at the time of admission or during the first 6 hours after ROSC) and need for persisted vasopressor support during TTM was significantly associated with poor outcome [32-34]. Although hypotension during TTM was not associated with poor neurological outcome in our study, any conclusions based on our results should be made with caution because the cause of hypotension is multifactorial and because persistent or severe hypotension during TTM is generally rare. 
Table 4 Factors associated with selected adverse events in univariate analyses

\begin{tabular}{|c|c|c|c|c|}
\hline Adverse event & Factors & OR & $(95 \% \mathrm{Cl})$ & $p$ \\
\hline \multirow[t]{3}{*}{ Bleeding } & Intra-aortic balloon pump & 5.40 & $(1.47-19.83)$ & 0.011 \\
\hline & Continuous renal replacement therapy & 3.13 & $(1.13-8.65)$ & 0.028 \\
\hline & Cardiogenic shock ${ }^{\mathrm{a}}$ & 2.39 & $(1.09-5.26)$ & 0.031 \\
\hline \multirow[t]{12}{*}{ Pneumonia $^{\mathrm{b}}$} & Ice bag only & 3.00 & $(1.79-5.02)$ & $<.001$ \\
\hline & Malignancy & 2.68 & $(1.04-6.90)$ & 0.041 \\
\hline & Neurological disease & 2.36 & $(1.25-4.46)$ & 0.008 \\
\hline & Non-shockable initial rhythm & 1.58 & $(1.14-2.20)$ & 0.006 \\
\hline & No bystander cardiopulmonary resuscitation & 1.45 & $(1.03-2.03)$ & 0.033 \\
\hline & Non-cardiac cause of arrest & 1.44 & $(1.06-1.96)$ & 0.021 \\
\hline & Initial presence of pupillary light reflex & 0.57 & $(0.42-0.78)$ & $<.001$ \\
\hline & ST-elevation myocardial infarction & 0.55 & $(0.31-0.98)$ & 0.042 \\
\hline & Coronary angiography & 0.51 & $(0.36-0.73)$ & $<.001$ \\
\hline & Angina pectoris & 0.49 & $(0.25-0.95)$ & 0.035 \\
\hline & Percutaneous coronary intervention & 0.47 & $(0.26-0.82)$ & 0.008 \\
\hline & Initial Glasgow coma scale $(\geq 5)$ & 0.44 & $(0.27-0.72)$ & 0.001 \\
\hline \multirow[t]{7}{*}{ Sepsis } & Intra-aortic balloon pump & 3.71 & $(1.43-9.67)$ & 0.007 \\
\hline & Ice bag only & 2.17 & $(1.20-3.94)$ & 0.010 \\
\hline & Unwitnessed arrest & 2.11 & $(1.37-3.24)$ & 0.001 \\
\hline & No bystander cardiopulmonary resuscitation & 1.78 & $(1.06-2.99)$ & 0.030 \\
\hline & Cardiogenic shock $^{\mathrm{a}}$ & 1.72 & $(1.10-2.68)$ & 0.017 \\
\hline & Non-cardiac cause of arrest & 1.65 & $(1.08-2.53)$ & 0.021 \\
\hline & Coronary angiography & 0.58 & $(0.35-0.98)$ & 0.040 \\
\hline \multirow[t]{4}{*}{ Myoclonus $^{c}$} & Ice bag only & 2.40 & $(1.42-4.05)$ & 0.001 \\
\hline & Hydrogel pad & 1.58 & $(1.05-2.38)$ & 0.027 \\
\hline & ST-elevation myocardial infarction & 0.36 & $(0.15-0.85)$ & 0.019 \\
\hline & Cooling garment & 0.06 & $(0.01-0.45)$ & 0.006 \\
\hline \multirow[t]{5}{*}{ Seizure $^{d}$} & Congestive heart failure & 2.68 & $(1.17-6.17)$ & 0.020 \\
\hline & Non-shockable initial rhythm & 1.51 & $(1.01-2.25)$ & 0.043 \\
\hline & Endovascular cooling & 0.64 & $(0.42-0.97)$ & 0.035 \\
\hline & Coronary angiography & 0.54 & $(0.35-0.83)$ & 0.005 \\
\hline & Initial Glasgow coma scale $\geq 5$ & 0.32 & $(0.16-0.65)$ & 0.002 \\
\hline \multirow[t]{7}{*}{ Hypoglycemia ${ }^{e}$} & Continuous renal replacement therapy & 2.59 & $(1.45-4.62)$ & 0.001 \\
\hline & Renal disease & 2.53 & $(1.32-4.84)$ & 0.005 \\
\hline & Non-shockable initial rhythm & 2.06 & $(1.37-3.11)$ & $<.001$ \\
\hline & Coronary angiography & 0.65 & $(0.43-0.98)$ & 0.039 \\
\hline & Hydrogel pad & 0.55 & $(0.34-0.88)$ & 0.013 \\
\hline & Percutaneous coronary intervention & 0.47 & $(0.23-0.98)$ & 0.042 \\
\hline & Cooling garment & 0.25 & $(0.09-0.70)$ & 0.008 \\
\hline
\end{tabular}

\footnotetext{
OR odds ratio, $95 \% \mathrm{Cl} 95 \%$ confidence interval
}

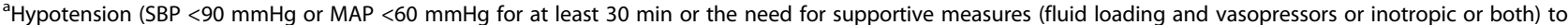
maintain a SBP $>90 \mathrm{mmHg}$ or MAP $>60 \mathrm{mmHg}$ ) and end-organ hypoperfusion (cool extremities)

${ }^{\mathrm{b}}$ Four requirements: 1) new or progressive consolidation on the chest radiograph, 2) fever, 3) leukocytosis, 4) presence of purulent tracheobronchial secretions 'Clinically involuntary movement without epileptiform discharge on EEG

${ }^{d}$ Either clinically involuntary movement with epileptiform discharge on EEG or epileptiform discharge only on EEG

$\mathrm{e} \leq 80 \mathrm{mg} / \mathrm{dL}$ within 72 hours after ROSC 
Overcooling was one of the most concerning AEs observed during the cooling period [35, 36]. Although overcooling during the cooling period was associated with poor neurological outcome based on univariate analysis, no such association was observed based on multivariate analysis in our study. The incidence (12\%) of rebound hyperthermia in our study was relatively lower than that in previous reports of post-hypothermia fever [37-40]. This discrepancy may be related to the differences in the definitions of temperature level, observation period and different populations between the compared studies. In our study, which defined post-hypothermia fever $\left(>38^{\circ} \mathrm{C}\right)$ within 24 hours of the cessation of cooling, rebound hyperthermia was not associated with poor neurological outcome based on either univariate or multivariate analysis. The influence of post-hypothermia fever on functional outcome is controversial [41, 42]. Therefore, further research on these temperature controlrelated AEs is necessary.

The incidence (39.6\%) of pneumonia during the advanced critical care period was similar to that described in most previous reports, including studies of cardiac arrest patients treated with TTM [7, 17, 18]. However, in some studies, the pneumonia incidence was higher than our result [11, 19, 43]. Differences in definitions, the use of prophylactic antibiotics, and the rate of WLST between studies may explain these discrepancies in the pneumonia incidence. In a recent report, prophylactic antibiotic use was associated with a lower incidence of pneumonia but did not appear to influence mortality or functional outcome in cardiac arrest survivors treated with TTM [44]. Because most participating hospitals in our registry used prophylactic antibiotics in their protocols, the incidence of pneumonia in our study may be lower than that in other studies $[11,19,43]$. However, the impact of prophylactic antibiotic use on functional outcome could not be evaluated because data concerning antibiotic use were not available in our registry. Thus, further studies are needed to investigate whether prophylactic antibiotic use impacts functional outcome.

The incidence (14\%) of sepsis during the advanced critical care period in our study was relatively higher than that in previous studies $[16,18,19]$. A retrospective study showed a similar incidence (13\%), and septic events had no impact on neurological outcome at hospital discharge [43]. In our study, after adjusting for independent predictors of poor neurological outcome, sepsis, but not pneumonia, was significantly associated with poor neurological outcome. In a recent systematic review and meta-analysis of TTM induced in adults for any indication, TTM was strongly associated with an increased risk of pneumonia and sepsis [45]. Most studies and meta-analyses including cardiac arrest patients showed that pneumonia and sepsis may be more frequent after TTM but that there were no significant differences in mortality or neurological outcomes between subjects undergoing TTM and normothermia or maintenance at $36{ }^{\circ} \mathrm{C}[11,16,17]$. In a prospective observational study including OHCA patients in Europe and North America, pneumonia and sepsis were inversely associated with mortality at 6 months based on univariate analysis [19]. The authors interpreted that the association of infections with mortality is possibly related to an increased risk of infection during a longer length of stay, which has been associated with a more favorable outcomes. However, pneumonia and sepsis were more frequent in patients with poor neurological outcome than in those with good neurological outcome in our study. The discrepancies between these two studies may be explained by the difference in the rate of WLST. The length of stay of the patients not awakening after sedation was typically longer than that of the patients who woke up after sedation was withdrawn because WLST was not performed in our study.

Hypokalemia and hyperglycemia during the cooling period were also frequently observed in our study, and these findings were consistent with those of previous reports [17-19]. Hypokalemia during the cooling period was not associated with poor neurological outcome, and this result was consistent with those of previous reports $[17,19]$. Hyperglycemia was associated with an increased incidence of poor outcome in patients who were not treated with TTM [46-48]. However, the influence of the blood glucose level during TTM and the advanced critical care period is uncertain. Sustained hyperglycemia ( $>8 \mathrm{mmol} / \mathrm{L}$ or $>144 \mathrm{mg} / \mathrm{dL}$ for $>4$ hours during the critical care period) was associated with increased mortality at 6 months in a prospective observational study of OHCA patients treated with TTM [19]. However, increased variability in the glucose level, but not the mean blood glucose level, during TTM was found to be an independent risk factor of in-hospital mortality in a prospective observational study [49]. In a recent large registry study that included OHCA patients treated with TTM, an increased median blood glucose level ( $>8.4 \mathrm{mmol} / \mathrm{L}$ or $>152 \mathrm{mg} / \mathrm{dL}$ ) over the first 48 hours was associated with poor neurological outcome [50]. In our study, hyperglycemia ( $>180 \mathrm{mg} / \mathrm{dL}$ ) during the cooling period was associated with poor neurological outcome based on univariate analysis but not multivariate analysis. Our results suggest that a single recording of a high blood glucose level during the cooling period may not be an independent risk factor of poor neurological outcome.

Notably, the incidence of hypoglycemia $(<4.4 \mathrm{mmol} / \mathrm{L}$ or $<80 \mathrm{mg} / \mathrm{dL}$ ) within 72 hours after ROSC was high (23\%) in our study and was significantly associated with 
poor neurological outcome at hospital discharge based on both univariate and multivariate analysis. These incidences were quite different from the results of the Target Temperature Management trial and an observational study (5.5\% and $5 \%$, respectively) in which hypoglycemia was defined at a lower level $(<3.0 \mathrm{mmol} / \mathrm{L}$ or $<54 \mathrm{mg} / \mathrm{dl}$ ) than our definition $[11,19]$. In the observational study, hypoglycemia during the critical care period was associated with increased mortality at 6 months based on univariate analysis but not multivariate analysis [19]. The reasons for the high incidence of hypoglycemia within 72 hours after ROSC in our study may be very complex. The lack of feeding, the use of intensive insulin therapy prior to the publication of the 2010 guidelines, and increased insulin sensitivity during rewarming may have influenced the high incidence of post-rewarming hypoglycemia within 72 hours after ROSC. Most hospitals that participated in our registry used no feeding protocol within 72 hours after ROSC because TTM may impair intestinal motility [15]. In a recent multicenter retrospective analysis, insulin sensitivity consistently increased over the first 36 hours [51]. Although the optimal level and duration of glycemic control during post-cardiac arrest care remains controversial, our results suggest that hypoglycemia within 72 hours after ROSC, even just below the normal range, may be harmful and may negatively impact neurological outcome because the injured brain is more sensitive to the glucose supply. Our results may support the recommendation of maintaining the blood glucose levels between 144 and $180 \mathrm{mg} / \mathrm{dL}$ based on the hypoglycemic risk of strict glucose control in the current guidelines $[8,9]$. Our findings suggest that the careful monitoring and prevention of hypoglycemia are important within 72 hours after ROSC.

The incidences of seizure and myoclonus during the advanced critical care period were high in our study and were similar to those of previous reports, which ranged from 15 to $44 \%$ [11, 19, 52, 53]. Seizure and myoclonus during the advanced critical care period were strongly associated with poor neurological outcome based on both univariate and multivariate analysis in our study, and these results were consistent with the results of previous studies [20, 54, 55]. Although anticonvulsant use was significantly associated with poor neurological outcome, this may not serve as an independent variable but rather may be collinear with seizure and myoclonus because most physicians in the participating hospitals in our registry only administered anticonvulsants when seizure or myoclonus was observed or highly suspected. The observed correlation between the use of anticonvulsants and poor outcome does not represent convincing evidence that this treatment contributes to poor outcome because patients with seizure after ROSC have likely experienced a major neurological injury.
Although seizure and myoclonus were strongly associated with poor neurological outcome, recent reports have suggested that some patients treated with TTM who experience seizure or myoclonus exhibit good neurological outcome $[19,56,57]$. In our study, $22 \%$ of patients with myoclonus, $16 \%$ of patients with seizure, and $25 \%$ of patients treated with anticonvulsants exhibited good neurological outcome at the time of hospital discharge. These results were similar to the result that $17 \%$ of OHCA patients with seizures exhibited good outcomes at 6 months in a previous observational study [19]. Therefore, careful monitoring and aggressive treatment of these AEs may be important for improving functional outcome.

An additional interesting finding in our study was the inverse association between $N M B$ use during TTM and poor neurological outcome at hospital discharge. This result may support the hypothesis of Salciccioli et al. [58], who reported that early continuous NMB use for a 24-hour period is associated with an increased probability of survival among post-cardiac arrest patients. In their study, NMB use showed a trend toward improved functional outcome, although this result was not statistically significant. Although we did not consider the NMB type, $\mathrm{NMB}$ delivery protocol, or duration of NMB administration, our result generates the following hypotheses for future research: patients with poor neurological outcome need less NMB use for shivering control, and NMB use during the early post-cardiac arrest care period may improve functional outcome.

\section{Limitations}

There were several limitations to our study. First, although we attempted to collect all consecutive OHCA patients who were treated with TTM in each hospital during the study period, there was an inevitable risk of selection bias because our study employed a retrospective design. Second, although we employed a standard investigation protocol, verified data completeness according to the site principle investigators, and used a team to perform data quality control, our study was subject to reporting bias because it was a registry-based multicenter study. Furthermore, the variability in TTM implementation timing and in the TTM protocol among hospitals may have impact the incidences of AEs and outcomes. Third, some of the AEs defined as other AEs, such as pneumonia and sepsis, may be considered cooling- or rewarming-related AEs. However, these AEs are difficult to diagnose during TTM and were recorded as other AEs during the advanced critical care period. Thus, our study cannot determine whether pneumonia or sepsis occurring during the cooling or rewarming period is associated with poor neurological outcome. Fourth, the data used in this study were collected from teaching hospitals in an Asian country; therefore, our results may have limited 
generalizability. Finally, we were only able to investigate neurological outcome at the time of hospital discharge, and this variable was not adequately blinded. This end point, which is not the current standard, may be not a reliable indicator of long-term outcome, although some investigators have suggested that the CPC score at the time of hospital discharge is a useful surrogate measure of long-term outcome [59].

\section{Conclusions}

In this retrospective study using multicenter registry data, cooling- and rewarming-related AEs were not associated with poor neurological outcome at the time of hospital discharge in OHCA patients treated with TTM. However, sepsis, myoclonus, seizure, hypoglycemia within 72 hours after ROSC, and the use of anticonvulsants during the advanced critical care period were significantly associated with poor neurological outcome at the time of hospital discharge in our study.

\section{Key messages}

- In this multicenter, registry-based study of adult OHCA patients treated with TTM in whom WLST was not performed, older age, previous poor health, a non-shockable initial rhythm, a longer time from arrest to ROSC, a non-cardiac cause of arrest, and CRRT were independent predictors of poor neurological outcome at the time of hospital discharge. Alternatively, initial GCS score $\geq 5$, initial presence of the pupillary light reflex, and coronary angiography were inversely associated with poor neurological outcome.

- After adjusting for independent predictors of poor neurological outcome, cooling- and rewarming-related AEs were not significantly associated with poor neurological outcome at the time of hospital discharge.

- After adjusting for independent predictors of poor neurological outcome, sepsis, myoclonus, seizure, hypoglycemia within 72 hours after ROSC, and the use of anticonvulsants during the advanced critical care period were significantly associated with poor neurological outcome at the time of hospital discharge.

\section{Additional files}

Additional file 1: KORHN registry case report form. Korean

Hypothermia Network (KORHN) registry case report form.

Additional file 2: KORHN registry investigator manual. Korean

Hypothermia Network (KORHN) registry investigator manual.

Additional file 3: Figure S1. Definition of the period in post-cardiac arrest care. Figure S2. Geographic distribution and the number of patients enrolled in the 24 participating centers.
Additional file 4: Sensitivity analysis II. Results of the sensitivity analysis for the patients who survived after sedation was withdrawn.

Additional file 5: The name of ethics bodies and site principal investigators in the participating hospitals.

\section{Abbreviations}

$95 \%$ Cl: 95 \% confidence interval; AE: adverse event; AUC: area under the receiver operating characteristic curve; CABG: coronary artery bypass graft; CPC: cerebral performance category; CPR: cardiopulmonary resuscitation; CRRT: continuous renal replacement therapy; ECMO: extracorporeal membrane oxygenation; ED: emergency department;

EEG: electroencephalography; EMS: emergency medical services; GCS: Glasgow coma scale; IABP: intra-aortic balloon pump; ICU: intensive care unit; MAP: mean arterial pressure; NMB: neuromuscular blocker; OHCA: out-of-hospital cardiac arrest; OR: odds ratio; PCl: percutaneous coronary intervention; ROSC: return of spontaneous circulation; SBP: systolic blood pressure; STEMI: ST-segment elevation myocardial infarction; TTM: targeted temperature management; WLST: withdrawal of life-sustaining treatment.

\section{Competing interests}

The authors declare that they have no competing interests.

\section{Authors' contributions}

YMK and KNP conceived the study. YMK, CSY, BKL, GCC, KWJ, and KCC designed the registry. CSY, SHK, BKL, ISC, GCC, KWJ, SHO, SPC, JHS, KCC, JSO, and KNP conducted data collection and managed the data, including quality control. YMK and HWY analyzed the data and interpreted the results. YMK drafted the manuscript, and all authors contributed substantially to its revision. KNP takes responsibility for the manuscript as a whole. All authors read and approved the manuscript.

\section{Acknowledgements}

We appreciate the help of all of the site investigators and clinical research coordinators in the participating hospitals with data input during the registry project. We also appreciate the statistical advice and critical manuscript revisions of Hyunyong Lee in the Clinical Research Coordinating Center at the Catholic Medical Center. The name of ethics bodies and site principal investigators in the participating hospitals are listed in Additional file 5.

\section{Funding}

This study was partially supported by a national research grant from the Korean Ministry of Health and Welfare in 2011.

\section{Author details}

'Department of Emergency Medicine, College of Medicine, The Catholic University of Korea, 222 Banpo-daero, Seocho-gu, Seoul 137-701, South Korea. ${ }^{2}$ Department of Emergency Medicine, College of Medicine, Chonnam National University, 160 Baekseo-ro, Dong-gu, Gwangju 501-746, South Korea. ${ }^{3}$ Department of Emergency Medicine, KEPCO Medical Center, 308 Uicheon-ro, Dobong-gu, Seoul 132-703, South Korea. ${ }^{4}$ Department of Emergency Medicine, College of Medicine, Hallym University, Kangdong Sacread Heart Hospital 150 Seongan-ro, Gangdong-gu, Seoul 134-701, South Korea. ${ }^{5}$ Department of Emergency Medicine, Boramae Medical Center, Seoul National University, 20 Boramae-ro, Dongjak-gu, Seoul 156-707, South Korea. ${ }^{6}$ Department of Emergency Medicine, Wonju College of Medicine, Yonsei University, 20 Ilsan-ro, Wonju, Gangwon-do 220-701, South Korea. ${ }^{7}$ Department of Preventive Medicine, College of Medicine, The Catholic University of Korea, 222 Banpo-daero, Seocho-gu, Seoul 137-701, South Korea.

Received: 8 April 2015 Accepted: 19 June 2015

Published online: 22 July 2015

\section{References}

1. Mozaffarian D, Benjamin EJ, Go AS, Arnett DK, Blaha MJ, Cushman M, et al. Heart disease and stroke statistics-2015 update: a report from the American Heart Association. Circulation. 2015;131:e29-e322.

2. Atwood C, Eisenberg MS, Herlitz J, Rea TD. Incidence of EMS-treated out-of hospital cardiac arrest in Europe. Resuscitation. 2005;67:75-80. 
3. Ro YS, Shin SD, Song KJ, Lee EJ, Kim JY, Ahn KO, et al. A trend in epidemiology and outcomes of out-of-hospital cardiac arrest by urbanization level: a nationwide observational study from 2006 to 2010 in South Korea. Resuscitation. 2013;84:547-57.

4. Laver S, Farrow C, Turner D, Nolan J. Mode of death after admission to an intensive care unit following cardiac arrest. Intensive Care Med. 2004;30:2126-8.

5. Dragancea I, Rundgren $M$, Englund $E$, Friberg $H_{1}$ Cronberg $T$. The influence of induced hypothermia and delayed prognostication on the mode of death after cardiac arrest. Resuscitation. 2013;84:337-42.

6. Bernard SA, Gray TW, Buist MD, Jones BM, Silvester W, Gutteridge G, et al. Treatment of comatose survivors of out-of-hospital cardiac arrest with induced hypothermia. N Engl J Med. 2002;346:557-63.

7. The Hypothermia after Cardiac Arrest Study Group. Mild therapeutic hypothermia to improve the neurologic outcome after cardiac arrest. N Engl J Med. 2002;346:549-56.

8. Peberdy MA, Callaway CW, Neumar RW, Geocadin RG, Zimmerman JL, Donnino M, et al. Part 9: post-cardiac arrest care: 2010 American Heart Association Guidelines for Cardiopulmonary Resuscitation and Emergency Cardiovascular Care. Circulation. 2010;122:S768-86.

9. Deakin CD, Nolan JP, Soar J, Sunde K, Koster RW, Smith GB, et al. European Resuscitation Council Guidelines for Resuscitation 2010 Section 4. Adult advanced life support. Resuscitation. 2010;81:1305-52.

10. Nunnally ME, Jaeschke R, Bellingan GJ, Lacroix J, Mourvillier B, Rodriguez-Vega GM, et al. Targeted temperature management in critical care: a report and recommendations from five professional societies. Crit Care Med. 2011;39:1113-25

11. Nielsen N, Wetterslev J, Cronberg T, Erlinge D, Gasche $Y$, Hassager C, et al. Targeted temperature management at $33^{\circ} \mathrm{C}$ versus $36^{\circ} \mathrm{C}$ after cardiac arrest. N Engl J Med. 2013;369:2197-206.

12. Cronberg T, Lilja G, Horn J, Kjaergaard J, Wise MP, Pellis T, et al. Neurologic function and health-related quality of life in patients following targeted temperature management at $33^{\circ} \mathrm{C}$ vs $36^{\circ} \mathrm{C}$ after out-of-hospital cardiac arrest: a randomized clinical trial. JAMA Neurol. 2015;72:634-41.

13. Lilja G, Nielsen N, Friberg H, Horn J, Kjaergaard J, Nilsson F, et al. Cognitive function in survivors of out-of-hospital cardiac arrest after target temperature management at $33^{\circ} \mathrm{C}$ versus $36^{\circ} \mathrm{C}$. Circulation. 2015:131:1340-9.

14. Polderman $\mathrm{KH}$, Herold I. Therapeutic hypothermia and controlled normothermia in the intensive care unit: practical considerations, side effects, and cooling methods. Crit Care Med. 2009;37:1101-20.

15. Polderman $\mathrm{KH}$. Application of therapeutic hypothermia in the intensive care unit. Opportunities and pitfalls of a promising treatment modality - Part 2. Practical aspects and side effects. Intensive Care Med. 2004;30:757-69.

16. Arrich J, Holzer M, Havel C. Hypothermia for neuroprotection in adults after cardiopulmonary resuscitation. Cochrane Database Syst Rev. 2012;9:CD004128.

17. Xiao G, Guo Q, Shu M, Xie X, Deng J, Zhu Y, et al. Safety profile and outcome of mild therapeutic hypothermia in patients following cardiac arrest: systematic review and meta-analysis. Emerg Med J. 2013;30:91-100.

18. Nielsen N, Hovdenes J, Nilsson F, Rubertsson S, Stammet P, Sunde K, et al. Outcome, timing and adverse events in therapeutic hypothermia after out-of-hospital cardiac arrest. Acta Anaesthesiol Scand. 2009;53:926-34.

19. Nielsen N, Sunde K, Hovdenes J, Riker RR, Rubertsson S, Stammet P, et al. Adverse events and their relation to mortality in out-of-hospital cardiac arrest patients treated with therapeutic hypothermia. Crit Care Med. 2011:39:57-64

20. MacLaren R, Gallagher J, Shin J, Varnado S, Nguyen L. Assessment of adverse events and predictors of neurological recovery after therapeutic hypothermia. Ann Pharmacother. 2014;48:17-25.

21. Jacobs I, Nadkarni V, Bahr J, Berg RA, Billi JE, Bossaert L, et al. Cardiac arrest and cardiopulmonary resuscitation outcome reports: update and simplification of the Utstein templates for resuscitation registries: a statement for healthcare professionals from a task force of the International Liaison Committee on Resuscitation. Circulation. 2004;110:3385-97.

22. Langhelle A, Nolan J, Herlitz J, Castren M, Wenzel V, Soreide E, et al. 2003 Utstein Consensus Symposium: Recommended guidelines for reviewing, reporting, and conducting research on post-resuscitation care: the Utstein style. Resuscitation. 2005;2003:271-83.

23. Byung Kook L, Kyu Nam P, Gu Hyun K, Kyung Hwan K, Giwoon K, Won Young K, et al. Outcome and current status of therapeutic hypothermia after out-of-hospital cardiac arrest in Korea using data from the Korea Hypothermia Network registry. Clin Exp Emerg Med. 2014;1:19-27.

24. Tsai MS, Chiang WC, Lee CC, Hsieh CC, Ko PC, Hsu CY, et al. Infections in the survivors of out-of-hospital cardiac arrest in the first 7 days. Intensive Care Med. 2005;31:621-6

25. Levy MM, Fink MP, Marshall JC, Abraham E, Angus D, Cook D, et al. 2001SCCM/ESICM/ACCP/ATS/SIS International Sepsis Definitions Conference. Crit Care Med. 2003;31:1250-6.

26. Jennett $B$, Bond M. Assessment of outcome after severe brain damage. Lancet. 1975;1:480-4.

27. Friberg H, Cronberg T, Dünser MW, Duranteau J, Horn J, Oddo M. Survey on current practices for neurological prognostication after cardiac arrest. Resuscitation. 2015;90:158-62

28. Stockmann H, Krannich A, Schroeder T, Storm C. Therapeutic temperature management after cardiac arrest and the risk of bleeding: systematic review and meta-analysis. Resuscitation. 2014;85:1494-503.

29. Stær-Jensen $H$, Sunde $K$, Olasveengen TM, Jacobsen D, Drægni $T$, Nakstad ER, et al. Bradycardia during therapeutic hypothermia is associated with good neurologic outcome in comatose survivors of out-of-hospital cardiac arrest. Crit Care Med. 2014:42:2401-8

30. Thomsen $\mathrm{JH}$, Hassager C, Bro-Jeppesen J, Søholm H, Nielsen $\mathrm{N}$, Wanscher $\mathrm{M}$, et al. Sinus bradycardia during hypothermia in comatose survivors of out-of-hospital cardiac arrest - a new early marker of favorable outcome? Resuscitation. 2015;89:36-42.

31. Torgersen C, Meichtry J, Schmittinger CA, Bloechlinger S, Jakob SM, Takala J, et al. Haemodynamic variables and functional outcome in hypothermic patients following out-of-hospital cardiac arrest. Resuscitation. 2013;84:798-804

32. Bro-Jeppesen J, Kjaergaard J, Søholm H, Wanscher M, Lippert FK, Møller JE, et al. Hemodynamics and vasopressor support in therapeutic hypothermia after cardiac arrest: prognostic implications. Resuscitation. 2014;85:664-70.

33. Beylin ME, Perman SM, Abella BS, Leary M, Shofer FS, Grossestreuer AV, et al Higher mean arterial pressure with or without vasoactive agents is associated with increased survival and better neurological outcomes in comatose survivors of cardiac arrest. Intensive Care Med. 2013;39:1981-8.

34. Bro-Jeppesen J, Annborn M, Hassager C, Wise MP, Pelosi P, Nielsen N, et al Hemodynamics and vasopressor support during targeted temperature management at $33{ }^{\circ} \mathrm{C}$ Versus $36^{\circ} \mathrm{C}$ after out-of-hospital cardiac arrest: a post hoc study of the target temperature management trial. Crit Care Med. 2015;43:318-27.

35. Merchant RM, Abella BS, Peberdy MA, Soar J, Ong ME, Schmidt GA, et al. Therapeutic hypothermia after cardiac arrest: unintentional overcooling is common using ice packs and conventional cooling blankets. Crit Care Med. 2006;34:S490-4.

36. Skulec R, Kovárník T, Bĕlohlávek J, Dostálová G, Kolár J, Linhart A, et al. Overcooling during mild hypothermia in cardiac arrest survivors-phenomenon we should keep in mind. Vnitr Lek. 2008;54:609-14.

37. Leary M, Grossestreuer AV, lannacone S, Gonzalez M, Shofer FS, Povey C, et al. Pyrexia and neurologic outcomes after therapeutic hypothermia for cardiac arrest. Resuscitation. 2013;84:1056-61.

38. Winters SA, Wolf KH, Kettinger SA, Seif EK, Jones JS, Bacon-Baguley T. Assessment of risk factors for post-rewarming "rebound hyperthermia" in cardiac arrest patients undergoing therapeutic hypothermia. Resuscitation. 2013;84:1245-9.

39. Cocchi MN, Boone MD, Giberson B, Giberson T, Farrell E, Salciccioli JD, et at. Fever after rewarming: incidence of pyrexia in post-cardiac arrest patients who have undergone mild therapeutic hypothermia. J Intensive Care Med. 2014:29:365-9.

40. Bro-Jeppesen J, Hassager C, Wanscher M, Søholm H, Thomsen JH, Lippert FK, et al. Post-hypothermia fever is associated with increased mortality after out-of-hospital cardiac arrest. Resuscitation. 2013;84:1734-40

41. Cronberg T, Nielsen N. Fever after therapeutic hypothermia-does rebound pyrexia matter? Resuscitation. 2013;84:1011-2.

42. Brugger $H$, Paal P. Does untreated post-cardiac-arrest fever counteract the benefit of therapeutic hypothermia? Resuscitation. 2013:84:1650-01.

43. Mongardon N, Perbet S, Lemiale V, Dumas F, Poupet $H$, Charpentier J, et al Infectious complications in out-of-hospital cardiac arrest patients in the therapeutic hypothermia era. Crit Care Med. 2011;39:1359-64

44. Gagnon DJ, Nielsen N, Fraser GL, Riker RR, Dziodzio J, Sunde K, et al. Prophylactic antibiotics are associated with a lower incidence of pneumonia 
in cardiac arrest survivors treated with targeted temperature management. Resuscitation. 2015;92:154-9.

45. Geurts M, Macleod MR, Kollmar R, Kremer PH, van der Worp HB. Therapeutic hypothermia and the risk of infection: a systematic review and meta-analysis. Crit Care Med. 2014;42:231-42.

46. Longstreth Jr WT, Diehr P, Cobb LA, Hanson RW, Blair AD. Neurologic outcome and blood glucose levels during out-of-hospital cardiopulmonary resuscitation. Neurology. 1986;36:1186-91.

47. Müllner M, Sterz F, Binder M, Schreiber W, Deimel A, Laggner AN. Blood glucose concentration after cardiopulmonary resuscitation influences functional neurological recovery in human cardiac arrest survivors. J Cereb Blood Flow Metab. 1997;17:430-6.

48. Langhelle A, Tyvold SS, Lexow K, Hapnes SA, Sunde K, Steen PA. Inhospital factors associated with improved outcome after out-of-hospital cardiac arrest. A comparison between four regions in Norway. Resuscitation. 2003;56:247-63.

49. Cueni-Villoz N, Devigili A, Delodder F, Cianferoni S, Feihl F, Rossetti AO, et al. Increased blood glucose variability during therapeutic hypothermia and outcome after cardiac arrest. Crit Care Med. 2011;39:2225-31.

50. Daviaud F, Dumas F, Demars N, Geri G, Bouglé A, Morichau-Beauchant T, et al. Blood glucose level and outcome after cardiac arrest: insights from a large registry in the hypothermia era. Intensive Care Med. 2014;40:855-62.

51. Sah Pri A, Chase JG, Pretty CG, Shaw GM, Preiser JC, Vincent JL, et al. Evolution of insulin sensitivity and its variability in out of hospital cardiac arrest (OHCA) patients treated with hypothermia. Crit Care. 2014;18:586.

52. Krumholz A, Stern BJ, Weiss HD. Outcome from coma after cardiopulmonary resuscitation: Relation to seizures and myoclonus. Neurology. 1988;38:401-5.

53. Mani R, Schmitt SE, Mazer M, Putt ME, Gaieski DF. The frequency and timing of epileptiform activity on continuous electroencephalogram in comatose post-cardiac arrest syndrome patients treated with therapeutic hypothermia. Resuscitation. 2012;83:840-7.

54. Hui AC, Cheng C, Lam A, Mok V, Joynt GM. Prognosis following postanoxic myoclonus status epilepticus. Eur Neurol. 2005;54:10-3.

55. Legriel S, Hilly-Ginoux J, Resche-Rigon M, Merceron S, Pinoteau J, Henry-Lagarrigue $\mathrm{M}$, et al. Prognostic value of electrographic postanoxic status epilepticus in comatose cardiac-arrest survivors in the therapeutic hypothermia era. Resuscitation. 2013;84:343-50.

56. Rossetti AO, Oddo M, Liaudet L, Kaplan PW. Predictors of awakening from postanoxic status epilepticus after therapeutic hypothermia. Neurology. 2009; $72: 744-9$

57. Lucas JM, Cocchi MN, Salciccioli J, Stanbridge JA, Geocadin RG, Herman ST, et al. Neurologic recovery after therapeutic hypothermia in patients with post-cardiac arrest myoclonus. Resuscitation. 2012;83:265-9.

58. Salciccioli JD, Cocchi MN, Rittenberger JC, Peberdy MA, Ornato JP, Abella BS, et al. Continuous neuromuscular blockade is associated with decreased mortality in post-cardiac arrest patients. Resuscitation. 2013;84:1728-33.

59. Phelps R, Dumas F, Maynard C, Silver J, Rea T. Cerebral Performance Category and long-term prognosis following out-of-hospital cardiac arrest. Crit Care Med. 2013;41:1252-7.

\section{Submit your next manuscript to BioMed Central and take full advantage of:}

- Convenient online submission

- Thorough peer review

- No space constraints or color figure charges

- Immediate publication on acceptance

- Inclusion in PubMed, CAS, Scopus and Google Scholar

- Research which is freely available for redistribution 